\title{
The Road Less Traveled: Integrating Neurotherapy with Holistic Neuropsychological Rehabilitation After Severe Head Injury
}

\author{
Mohammed Afsar, Nishita Choudhari, Dhaval Shukla, and Jamuna Rajeswaran*
}

National Institute of Mental Health and Neuro Sciences, Bangalore, India

\begin{abstract}
Introduction: Traumatic brain injury (TBI) is associated with physical, cognitive, emotional, and behavioral disturbances. The cognitive deficits are common after TBI, and a holistic approach to neuropsychological rehabilitation is recommended in these patients. EEG neurofeedback training (EEG-NFT) is a state-of-the-art technique for neuropsychological rehabilitation. There is a paucity of studies exploring the use of EEG-NFT integrated with holistic neuropsychological rehabilitation. Method: Single case design was adopted for the present study. A 25-year-old single male, diagnosed with severe TBI, presented with physical, cognitive, and emotional-behavioral disturbances after 17 months of injury. A comprehensive neuropsychological assessment was carried out. The neuropsychological rehabilitation using EEG-NFT along with psychosocial interventions with the patient and the parents was carried out for 9 months. Results: The patient showed significant improvement in cognitive deficits such as attention, executive functions, and visuospatial ability. Emotional-behavioral problems such as irritability, sadness, and overall dysfunction also improved significantly. Conclusion: The present case study highlights that integrating EEG-NFT along with holistic neuropsychological rehabilitation helps to improve cognitive, emotional, and behavioral disturbances after TBI.
\end{abstract}

Keywords: EEG neurofeedback training; neuropsychological rehabilitation; holistic neuropsychological rehabilitation; severe traumatic brain injury; neurotherapy

Citation: Afsar, M., Choudhari, N., Shukla, D., \& Rajeswaran J. (2021). The road less traveled: Integrating neurotherapy with holistic neuropsychological rehabilitation after severe head injury. NeuroRegulation, 8(1), 57-68. https://doi.org/10.15540/nr.8.1.57

*Address correspondence to: Dr. Jamuna Rajeswaran, MPhil, $\mathrm{PhD}$, Professor, Department of Clinical Psychology, 3rd Floor, M V Govindaswamy Centre National Institute of Mental Health Neuro Sciences (NIMHANS), Bangalore, India 560029. Email: drjamunarajan@gmail.com

Copyright: (c) 2021. Afsar et al. This is an Open Access article distributed under the terms of the Creative Commons Attribution License (CC-BY).

\section{Edited by:}

Rex L. Cannon, PhD, SPESA Research Institute, Knoxville, Tennessee, USA

\section{Reviewed by:}

Rex L. Cannon, PhD, SPESA Research Institute, Knoxville, Tennessee, USA

Randall Lyle, PhD, Mount Mercy University, Cedar Rapids, lowa, USA

\section{Introduction}

Traumatic brain injury (TBI) is a major public health concern in India. TBI not only affects the individual but it also affects the family, society, and economy at large. The rapid increase in population over the last few decades along with exponential economic growth, particularly in motorization and industrialization, has contributed to a significant increase in TBI over the years. TBI has been defined as "an alteration in brain function, or other evidence of brain pathology, caused by external force" (Menon, Schwab, Wright, \& Maas, 2010). As a syndrome, it is heterogeneous in its pathophysiology and is therefore classified in terms of differing levels for severity (i.e., mild, moderate and severe) for better understanding and management (Sternbach, 2000). The Glasgow Coma Scale (GCS) is the most widely used instrument to assess the severity of TBI. A score of 8 or less after resuscitation, within 48 hours of injury is considered to represent a severe TBI (Foulkes, Eisenberg, Jane, Marmarou, \& Marshall, 1991). The data across the globe shows that around $81 \%$ of all TBls are of mild severity (Dewan et al., 2018). The impact of TBI is reflected in a wide range of physical, cognitive, emotional, and behavioral disturbances. The survivors of $\mathrm{TBI}$, especially with moderate or severe TBIs, often suffer from chronic disability (Burton, 2016). 


\section{Posttraumatic Sequelae}

Cognitive deficits remain at the core of problems experienced by survivors of $\mathrm{TBI}$ also known as posttraumatic sequelae. Studies in survivors of TBI show impairment in attention (Dikmen et al., 2009; Leclercq et al., 2000; McAvinue, O'Keeffe, McMackin, \& Robertson, 2005; Reddy, Rajeswaran, Devi, \& Kandavel, 2017) and speed of information processing (Madigan, DeLuca, Diamond, Tramontano, \& Averill, 2000; Rassovsky et al., 2006). Deficits in complex executive functions, such as reasoning, planning, problem-solving, emotional self-regulation, decision-making, and judgment have also been reported (Dikmen et al., 2009; Rabinowitz \& Lewin, 2014; Serino et al., 2006) along with impairment in memory function, predominantly in the encoding and retrieval aspects of memory (Dikmen, Machamer, Powell, \& Temkin, 2003; Kersel, Marsh, Havill, \& Sleigh, 2001; Rabinowitz \& Levin, 2014).

Behavioral disturbances are commonly seen after TBI and often exist simultaneously along with cognitive deficits. These behavioral disturbances are seen in the form of apathy, disinhibition, agitation, aggression, and violent behavior (Rabinowitz \& Levin, 2014; Stéfan \& Mathé, 2016). About $50-60 \%$ of patients with severe TBI often exhibit these disturbances and have difficulties impacting multiple domains of functioning such as occupation, interpersonal relationships, and sexual functioning. Depression and anxiety are the most commonly reported emotional disturbances after TBI and can negatively affect the long term outcomes (Albrecht, Barbour, Abariga, Rao, \& Perfetto, 2019; Scholten et al., 2016; War, Jamuna, \& Arivazhagan, 2014). The loss of employment secondary to TBI can have further cascading effects on finances, interpersonal relationships, and quality of life (Ahmed et al., 2017; Benedictus, Spikman, \& van der Naalt, 2010). Thus, as a result of these neuropsychiatric complications, several patients are left without the ability to work or to perform activities of daily living.

\section{Neuropsychological Rehabilitation}

Due to the multitude of impairments in the domains of cognition, emotion, and behavior, the neuropsychological rehabilitation of a person with TBI poses a unique challenge. Neuropsychological rehabilitation is defined as a process concerned with the amelioration of cognitive, emotional, psychosocial, and behavioral deficits caused by an insult to the brain (Wilson, 2008). Neural plasticity, the main principle underlying neuropsychological rehabilitation, refers to the ability of the nervous system to adapt itself, structurally and functionally, in response to experience and injury (von Bernhardi, Eugenín-von Bernhardi, \& Eugenín, 2017). There are different forms of neuropsychological rehabilitation such as cognitive retraining, computerbased retraining, EEG-neurofeedback training (EEG-NFT). A holistic approach to neuropsychological rehabilitation is recommended which not only addresses the cognitive impairments but also equally emphasize on the emotionalbehavioral, sexual, and vocational issues (Rajeswaran, 2012).

EEG-neurofeedback training (EEG-NFT) is a relatively newer neuroscience-based intervention modality, which is increasingly being used for in a wide range of clinical conditions. EEG-NFT works on the principles of operant conditioning and is deemed to be potent to modify the structural and functional abnormalities in cortico-cortical and cortico-subcortical neural networks (Munivenkatappa, Rajeswaren, Indira Devi, Bennet, \& Upadhyay, 2014). Single case studies and case series of EEG-NFT in patients with TBI showed improvements in executive functions, learning and memory, anosmia, agraphia, anxiety, and depressive symptoms (Bennett, Rajeswaran, Sampath, \& Christopher, 2013; Byers, 1995; Hammond, 2007; Reddy, Jamuna, Indira Devi, \& Thennarasu, 2009; Thornton, 2000). The studies with larger samples have replicated these results. Another study, by Zorcec, Demerdzieva, and PopJordanova (2011) examined the effects of EEG-NFT in six patients with TBI. Following 20 sessions of EEG-NFT, five of the six patients were able to return to normal education, and all reported improvements in mood, sleep, and cognition. May, Benson, Balon, and Boutros (2013) conducted a review of published studies pertaining to the utility of EEG-NFT in patients with TBI. They concluded that EEG-NFT is probably effective in the rehabilitation after TBI; however, the heterogeneity of the syndrome poses a big challenge to draw any strong inferences from the available body of literature.

The beginning of the 21st century marked the introduction of EEG-NFT in India as a method of neuropsychological rehabilitation. Since then, many scientific investigations using EEG-NFT have been carried out. Twenty sessions of EEG-NFT, each lasting for $40 \mathrm{~min}$ on alternate days with a protocol targeting to increase alpha and decrease theta resulted in significant improvements in a wide range of cognitive functions such as processing speed, attention, executive functions, visuospatial construction, learning, and memory in patients with TBI (Reddy, Rajeswaran, Indira Devi, \& Kandavel, 
2013). EEG-NFT was also found to be useful in improving subjective symptom reporting, perceived stress, and quality of life (Bennett et al., 2018; Bennett, Sampath, Christopher, Thennarasu, \& Rajeswaran, 2017; Reddy, Rajeswaran, Bhagavatula, \& Kandavel, 2014). These findings suggest that EEG-NFT can lead to dual benefit (i.e., by bringing about improvement in cognitive functions as well as in emotional-behavioral functioning).

EEG-NFT is used extensively in neuropsychological rehabilitation after TBI. There are recent studies attempting to unravel the mechanism of EEG-NFT. Munivenkatappa et al. (2014) reported a significant increase in the cortical grey matter volume, enhanced white matter integrity, and increased global functional connectivity in two patients with severe TBI who received 20 sessions of EEG-NFT. Another recent study had shown that 20 sessions of EEG-NFT resulted in reduced postconcussion symptoms, reduced delta-alpha, and increased theta-alpha ratio on quantitative EEG (Gupta, Afsar, Yadav, Shukla, \& Rajeswaran, 2020). These findings indicate that EEG-NFT can bring about changes in neural functioning and thereby result in clinical improvement.

The published literature indicates positive effects of EEG-NFT in the improvement of both subjective symptom reporting and objective measures of neuropsychological functioning in TBI; however, the findings are primarily limited to mild and moderate TBI. More studies are needed to establish its effectiveness in patients with severe TBI. The current literature has shown the usefulness of holistic neuropsychological rehabilitation after TBI (Ben-Yishay \& Diller, 2011; Cicerone et al., 2008). However, most of the studies exploring the effectiveness of EEG-NFT in TBI have taken EEGNFT as a standalone technique of neuropsychological rehabilitation. It is widely agreed that a condition such as TBI results in disturbance in psychological and emotional functioning of the individual and also brings about significant distress and burnout in caregivers (Ahmed et al., 2017; Qadeer et al., 2017). There is a paucity of literature on the use of EEG-NFT in conjunction with holistic neuropsychological rehabilitation. Therefore, the present case study attempts to explore the role of EEG-NFT integrated neuropsychological rehabilitation in a patient with severe TBI.

\section{Methods}

The present study adopted a longitudinal case study design with repeated measurements over time.
Verbal and written informed consent were obtained from the patient as well as family members.

\section{Case Details}

Mr. $A B$ is a 25-year-old single male, who was pursuing an MBA after completing his graduation in commerce. He was apparently well until he was involved in a road traffic accident while riding his bike without a helmet in August 2017. The details of the accident were not known as there were no bystanders. He was found unconscious by other people on the road and rushed to the local medical center. He had bleeding from his mouth and left ear. There was no known history of seizure or vomiting. After an initial evaluation and life support, he was shifted to a neurosurgical facility for further evaluation and management. On arrival to the neurosurgery emergency, his GCS score was reported to be $E_{1} V_{1} M_{4}(06 / 15)$, suggestive of severe TBI. A CT scan of the brain was performed immediately, and it revealed fracture of the left ramus of the mandible and left temporal bone extending to sphenoid and odontoid processes. There was evidence of bleeding in cerebral cisterns. These symptoms were managed medically. An MRI of the brain done after 10 days of injury revealed multifocal acute onset hemorrhagic contusions in the left fronto-temporal cortical region, splenium and rostrum of corpus callosum, left side of midbrain, and upper part of the pons. There was further evidence of left fronto-temporo-parietal subdural hematoma with intraventricular haemorrhage. $\mathrm{He}$ was diagnosed with severe TBI with diffuse axonal injury (DAI) Grade III. The patient had posttraumatic amnesia for 3 months postinjury.

He was discharged after 2 months and had been undergoing physiotherapy and occupational therapy since discharge. $\mathrm{He}$ was showing gradual improvement. The patient presented to our center in February 2019 after 17 months of TBI. At the time of presentation, the chief complaints were rapid forgetting (forgetting recent conversations and what he had eaten for the last meal, not being able to remember the name of the therapist despite meeting him on a daily basis), behavioural changes in the form of impulsivity, poor frustration tolerance, irritability, and anger outbursts. His parents also reported that he had frequent crying spells, would often express death wishes, and had a negative view of self. These disturbances were secondary to his cognitive deficits and were often momentary in nature. He also had partial vision loss in the right visual field and mild residual weakness in his right half of the body. These complaints were being managed through an ophthalmology consultation 
and physiotherapy. Furthermore, he had speech and language-related issues such as difficulty naming objects, along with mild slurring of speech.

During the initial clinical evaluation, it was noticed that Mr. AB would get impatient frequently and would keep leaving the evaluation room. A lability in affect was noticed, with short periods of irritability seen several times during the session. He would be keen on performing certain tasks during the assessment but would lose interest quickly. When unable to recall certain things and events, he would keep responding "I don't know" and would ask the clinician to ask his parents instead. There was evidence of spontaneous confabulation, in terms of coming up with approximate answers to fill in the memory gaps. Further attempts to coax him to respond to these questions would be reciprocated with irritability. He reported feeling sad and having occasional death wishes. These complaints were associated with the termination of a longstanding romantic relationship, a sudden discontinuation of his education, and a sense of being incapacitated due to the TBI. He spoke loudly and his speech was mildly slurred. He would keep interrupting the therapist during the initial interview and assessment and would become restless quickly.

A comprehensive neuropsychological assessment (NPA) was carried out using NIMHANS neuropsychological battery for head injury (Rao, Subbakrishna, \& Gopukumar, 2004). The NIMHANS neuropsychological battery for head injury is a standardized neuropsychological battery that has been validated and widely used in the Indian population. Age and education matched norms are used for interpretation of the scores. The battery evaluates a range of cognitive domains including speed of information processing, focused attention, semantic fluency, working memory, planning, response inhibition, visuospatial construction, learning, and memory. These domains are assessed using eight different tests. The scores obtained are converted into percentiles using the normative data. The variables with a corresponding percentile of 15 or below are considered to be in impaired range. The visual analogue scale (VAS) was also used to assess the caregiver's impression of the level of emotional and behavioral disturbances in the patient. In VAS, caregivers are asked to rate the severity of symptoms or concern observed by them on a scale of zero to ten (0-10), where higher scores reflect more severe problems.

The baseline NPA (Test-1) indicated impairment in the cognitive domains processing speed, focused attention, working memory, semantic fluency, response inhibition, visual-spatial construction, and learning and memory (both verbal and visual). The VAS ratings were obtained from the father and showed very severe problems of forgetfulness and disorientation, followed by irritability. The overall dysfunction rating was $40 \%$. The findings from the baseline as well as follow-up NPA are given in Table 1.

During the process of initial evaluation and baseline assessments, it was found that there was significant helplessness and burnout present in the patient's parents. The parents also lacked understanding about the patient's condition, which consequently led to high expectations about recovery. Furthermore, his demanding and impulsive behavior, in conjunction with the parents' poor knowledge about illness, resulted in the overinvolvement of the parents in terms of carrying out activities of daily living for him, restricting his social interaction, and easily giving into his demands. It is noteworthy that even before the injury there was a significant amount of overinvolvement by the mother with the patient's upbringing, as he is a single child. It was hypothesized that this long-standing parental overprotection, combined with postinjury deficits, had resulted in the patient assuming a sick role and poor motivation to actively participate in the rehabilitation. The impact of the patient's TBI had caused a significant amount of emotional distress to the mother, who reported frequent crying spells and other depressive symptoms.

On the basis of the clinical data gathered from multiple sources, such as the review of previous records, initial interview, and neuropsychological assessment, the overall dysfunction experienced by Mr. AB was formulated using a model of postconcussive symptoms (Yeates, 2010), which is presented in Figure 1. 


\section{Table 1}

Findings from neuropsychological assessment (NPA) and visual analogue scale (VAS) at various time points.

\begin{tabular}{|c|c|c|c|c|c|}
\hline Functions & Tests & $\begin{array}{c}\text { Test-1 } \\
\text { 02/01/2019 }\end{array}$ & $\begin{array}{c}\text { Test-2 } \\
03 / 21 / 2019\end{array}$ & $\begin{array}{c}\text { Test-3 } \\
\text { 07/02/2019 }\end{array}$ & $\begin{array}{c}\text { Test-4 } \\
11 / 19 / 2019\end{array}$ \\
\hline Mental Speed & Digit Symbol Test & 5 & 8 & 8 & 21 \\
\hline \multirow{2}{*}{ Focused Attention } & Color Trails Test - 1 & 10 & 64 & 30 & 27 \\
\hline & Color Trails Test - 2 & 49 & 35 & 49 & 30 \\
\hline Semantic Fluency & Animals Names Test & 3 & 13 & 18 & 10 \\
\hline Working Memory & Spatial Span Test & 40 & 23 & 23 & 75 \\
\hline Response Inhibition & Stroop Test & Not Done* & 2 & 5 & 64 \\
\hline Planning & Tower of London & 90 & 94 & 80 & 90 \\
\hline $\begin{array}{l}\text { Visual-Spatial } \\
\text { Construction }\end{array}$ & CFT - Copy & 3 & 10 & 5 & 55 \\
\hline \multirow{2}{*}{ Visual Memory } & CFT - Immediate Recall & 3 & 3 & 3 & 3 \\
\hline & CFT - Delayed Recall & 3 & 3 & 3 & 3 \\
\hline \multirow{5}{*}{$\begin{array}{l}\text { Verbal Learning } \\
\text { and Memory }\end{array}$} & AVLT - Total Learning & 3 & 3 & 3 & 3 \\
\hline & AVLT - Immediate Recall & 3 & 3 & 3 & 3 \\
\hline & AVLT - Delayed Recall & 3 & 3 & 3 & 3 \\
\hline & AVLT - \% Retention & 3 & 3 & 3 & 14 \\
\hline & AVLT - Recognition (Hits) & 3 & 3 & 20 & 25 \\
\hline \multirow{6}{*}{$\begin{array}{l}\text { Subjective Report } \\
\text { of Caregivers }\end{array}$} & VAS - Forgetfulness & 9 & 7 & 6 & 6 \\
\hline & VAS - Irritability & 6 & 4 & 3 & 1 \\
\hline & VAS - Sadness & 7 & 6 & 7 & 5 \\
\hline & VAS - Speech & 3 & 2 & 1 & 1 \\
\hline & VAS - Disorientation & 9 & 6 & 5 & 2 \\
\hline & VAS - Overall Deficits & $40 \%$ & $25 \%$ & $20 \%$ & $15 \%$ \\
\hline
\end{tabular}

Note. ${ }^{*}=$ The test was attempted but had to be discontinued as the patient was finding it extremely difficult to name the colors; CFT - Complex figure test; AVLT - Auditory verbal learning test; VAS - Visual analogue scale. 


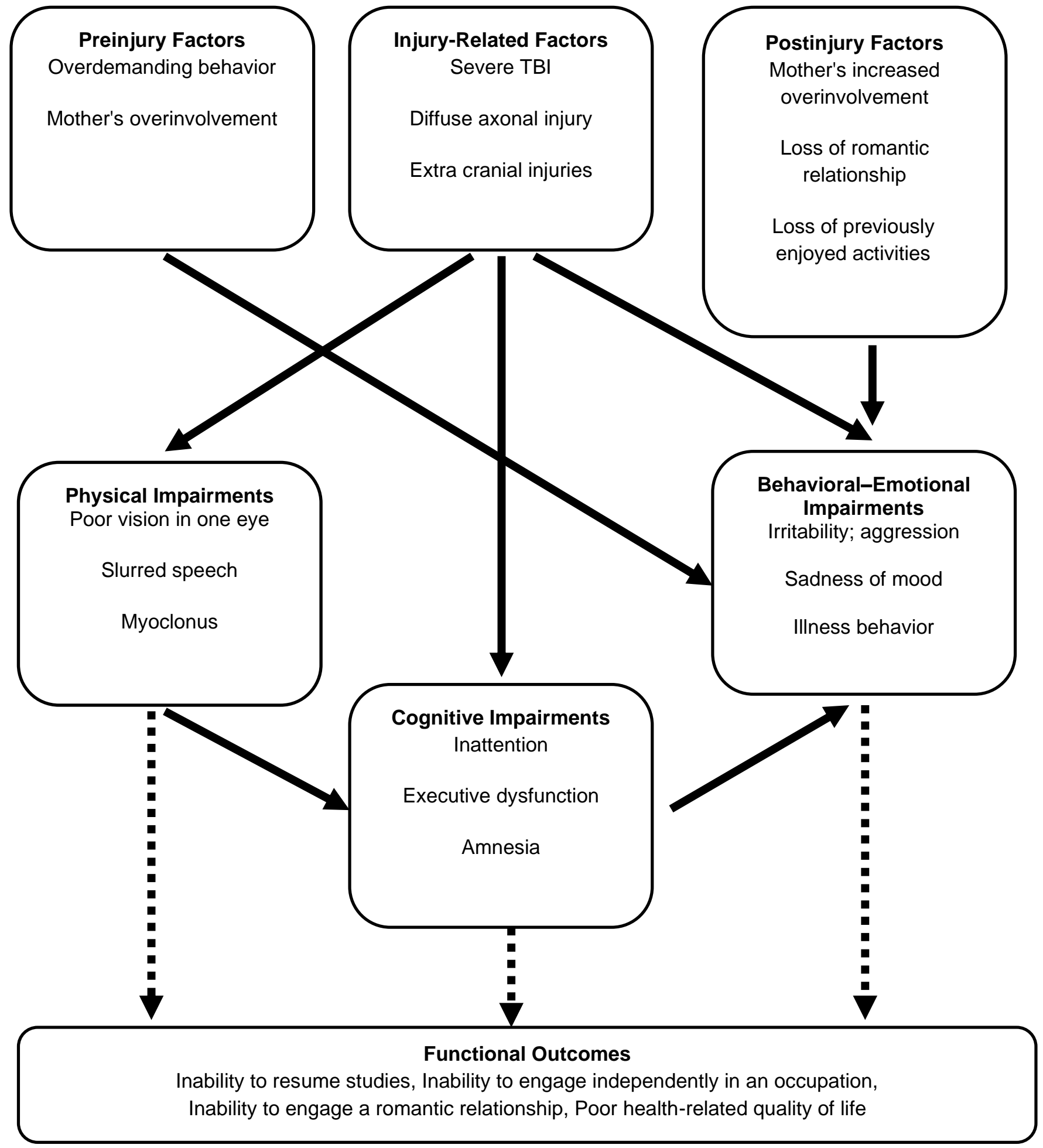

Figure 1. An integrated formulation of Mr. AB's posttraumatic symptoms. 
With an all-encompassing formulation of the case in mind, the neuropsychological rehabilitation was designed using a holistic approach. It was hypothesized that, to reduce overall dysfunction in him, all of the contributing factors need to be addressed. Therefore, the neuropsychological rehabilitation aimed at the following goals: (1) enhancing his cognitive functioning, (2) managing the behavioral and emotional disturbances, and (3) optimizing parental expectations and reducing their overinvolvement.

\section{EEG-Neurofeedback Training (EEG-NFT)}

As EEG-NFT has been found to be effective in reducing the cognitive, emotional, and behavioral problems after TBI, therefore it was adopted as the technique of neuropsychological rehabilitation. Patient received 20 sessions of EEG-NFT three times per week. The training protocol was a twochannel training provided at occipital scalp locations $\left(\mathrm{O}_{1}\right.$ and $\left.\mathrm{O}_{2}\right)$ in accordance with $10-20$ system and targeted at increasing the alpha power and decreasing theta power. The EEG-NFT was provided using a dedicated EEG-NFT system (Atlantis system, BrainMaster Technologies, Inc., Bedford, OH). The intervention sessions were comprised of a computer simulation wherein the patient was asked to relax and focus on the screen. He was provided feedback about his performance using auditory modality (a beep tone) and visual modality (scores on screen). Initially, it was observed that $\mathrm{Mr}$. $\mathrm{AB}$ would be very impatient during the EEG-NFT sessions. He would refuse to sit in one place any longer and would not be sufficiently engaged to focus on the task. A considerable amount of effort was invested into getting him to sit in one place and focus. Therefore, the sessions were initially carried out for a period of $20 \mathrm{~min}$ and, with an increase in the patient's sitting tolerance, the duration of the sessions were progressively increased to 40 min over a period of time.

\section{Psychotherapeutic Interventions}

With the patient. Supportive interventions, at a frequency of two sessions per week, were used to address the grief due to the termination of his previous relationship, the abrupt break in his education, and the restriction on performing activities he enjoyed previously (e.g., riding his motorcycle, going to the gym). The techniques used included emotional catharsis, guidance, reassurance, encouragement, and externalization of interests. Later sessions focused on collaboratively working on smaller, realistic goals, such as helping out in day-to-day chores, helping out his parents, keeping the appointment diary for his sessions, and recording his scores in the EEG-NFT. Behavioral techniques such as activity scheduling were used to implement these goals, and external aids such as using cued recall strategies were used to facilitate recall of recent events.

With the parents. In view of their poor knowledge about the patient's illness and parental overinvolvement, a supportive-educative approach to interventions was used to work with the parents. The sessions were carried out on a weekly basis for 7 consecutive weeks with both parents (Blake, 2008). The major themes of these sessions were feedback of test findings and its practical implications in daily life; shifting the focus from symptoms to functionality, neuroplasticity, and the need for neuropsychological rehabilitation; establishing practical and feasible goals for rehabilitation; and the need for developing autonomy and self-efficacy in the patient. Mr. AB's mother had symptoms of low mood and crying spells; she was referred for a psychiatric evaluation. She was diagnosed with an adjustment disorder and was started on antidepressant medications.

\section{Results}

The baselines assessments were repeated at the end of intervention. The postintervention NPA (Test-2) revealed that there were improvements noticed in processing speed, focused attention, semantic fluency, response inhibition, and visualspatial construction. There was no significant change observed on the scores of tests assessing learning and memory. However, the performance was still in the impaired range in most of the cognitive domains assessed. The VAS showed that his orientation improved significantly. There was also improvement noticed in reduced forgetfulness and irritability. Overall dysfunction came down to $25 \%$. The patient was sent back to home with advice to continue behavioral techniques and strategies at home and to follow up after 3 months.

The patient presented after 3 months for follow-up evaluation. Follow-up NPA (Test-3) showed that his scores on most of the cognitive tests remained unchanged, but a significant drop was noted on the tests of focused attention and planning. His father also reported only slight improvement in behavioral and emotional symptoms. The patient was offered EEG-NFT but, due to logistic reasons, the family could not stay back and therefore a home-based cognitive retraining program was provided. 


\section{Cognitive Retraining (CR)}

The home-based cognitive retraining $(\mathrm{CR})$ program was developed specifically for patients with head injury by Dr. Shobhini Rao in 2004 (Rao, 2004). The home-based CR intervention is based on the principles of restoration and aimed at enhancement of impaired cognitive functions through intensive and repetitive practice and drills. The $\mathrm{CR}$ intervention tasks targeted sustained attention, response inhibition, fluency, and working memory. These tasks included grain sorting, letter cancellation, shading, mental calculation, and word generation. The difficulty level for each of the tasks was gradually increased. All of the tasks were demonstrated to the parents in order to make them understand the administration and recording. They were advised to come back for EEG-NFT at their convenience.

Patient came back for follow-up after 3 months. Further 20 sessions of EEF-NFT were carried out using the same training protocol. Significant positive behavioral changes were observed such as reduced irritability, using requests rather than orders, reduced incidents of walk-outs from the EEG-NFT room, greater efforts for recollecting information, and overall cheerful mood.

At the end of 20 sessions a postintervention NPA was carried out. The NPA (Test-4) revealed that most of the cognitive domains (processing, speed, focussed attention, working memory, response inhibition, visual-spatial construction) had significantly improved and were no more in the impaired range of functioning. His scores (percentile ranks) on memory tests remains impaired, however; qualitatively, there was an improvement seen on raw scores on memory tests. On VAS, his father reported a significant improvement in orientation, irritability, and sadness. There was no change reported in his speech or forgetfulness. Overall dysfunction came down to $15 \%$.

\section{Discussion}

The present case study investigates the effects of EEG-NFT integrated with holistic neuropsychological rehabilitation over a period of one year in a patient with a severe TBI. The patient presented with a multitude of problems including physical, emotional, cognitive, and behavioral problems. A comprehensive neuropsychological assessment (NPA) battery and the visual analogue scale (VAS) were administered. The plan for neuropsychological rehabilitation was formulated based on a comprehensive model of postconcussive symptoms
(Yeates, 2010). EEG-NFT with the alpha-up, thetadown protocol was selected as a mainstay of neuropsychological rehabilitation and other modalities such as psychotherapy, family interventions, and cognitive retraining exercises were used to augment the EEG-NFT whenever necessary.

During the first phase of neuropsychological rehabilitation, EEG-NFT, psychotherapy, and family interventions were used. A follow-up assessment reflected significant improvement in processing speed, focused attention, semantic fluency, response inhibition, and visual-spatial construction. The scores on learning and memory, however, remained status quo. These findings are in line with the previous studies where EEG-NFT resulted in enhanced attention and response accuracy in a group of patients with mild TBI (Tinius \& Tinius, 2000). Similar findings were also observed in patients with moderate TBI where EEG-NFT led to enhanced accuracy in a processing speed test and faster reaction time in a sustained attention task (Keller, 2001). Another study from the southern Indian peninsula reported similar findings where 20 sessions of EEG-NFT with a similar alpha-theta protocol were effective in improving various cognitive domains in patients with mild to severe TBI (Reddy et al., 2013).

Unchanged scores on memory tests could be explained by two factors. Firstly, memory deficits after acquired brain injury have been found to be difficult to ameliorate with cognitive rehabilitation (Doornhein \& De Haan, 1998; Majid, Lincoln, \& Weyman, 2000). Secondly, the patient had severe TBI with a Grade III DAI, rendering the patient to have very severe memory deficits, thereby making the recovery of memory functions even more difficult.

One of the major challenges in the present case was the overlay of cognitive and emotional disturbances in the patient. Such overlay is common in clinical practice. It has been seen through literature that physical, behavioral, and cognitive symptoms are the primary targets in neuropsychological rehabilitation, whereas psychological and emotional issues often remain neglected (Bédard et al., 2012). Cognitive disturbances have a major impact on the patient's progress in psychosocial interventions for emotional problems whereas the emotionalbehavioral disturbances may block the pathway to cognitive recovery (Cole et al., 2015). It is recommended that a holistic neuropsychological rehabilitation program integrating the individualized 
interventions to target cognitive and interpersonal functioning should be provided to patients with TBI (Cicerone et al., 2019). Therefore, other psychotherapeutic interventions were also used along with EEG-NFT. A supportive-educative psychotherapeutic approach with use of cognitive behavioral techniques was used to work with the patient and the family.

The postassessment at the end of the first phase further revealed that there were significant changes in emotional and behavioral domains as seen on VAS scores. The improvements were reported in the problem areas of forgetfulness, irritability, and orientation. The overall dysfunction rating reduced from $40 \%$ to $25 \%$. These improvements can possibly be attributed to a combination of EEG-NFT and psychotherapeutic interventions. Similar findings have been reported previously in other Indian studies. Twenty sessions of EEG-NFT were effective in reducing the postconcussive symptoms, perceived stress, and overall levels of dysfunctions (Bennett et al., 2017; Reddy et al., 2014). It was also found that EEG-NFT was equally effective in bringing about these changes regardless of the time since injury (Bennett et al., 2017). Memory scores on neuropsychological testing did not show any change, but forgetfulness and orientation were reported to have improved on the VAS. It is likely that the micro improvements in memory were not sufficient enough to be reflected in the change in percentile ranks on memory tests. Psychological interventions with the parents resulted in the reduction of stress experienced by them at the end of phase I. Given the lack of adequate facilities available to the patient at his hometown and the lack of feasibility of more EEG-NFT sessions, the patient was discharged to home with the recommendation to actively engage in routine indoor and outdoor activities.

A follow-up assessment completed after 3 months of rehabilitation revealed that, although his performance was stable across most cognitive domains, there was a significant drop in his performance on focused attention and planning. The VAS also indicated only minimal improvement in the emotional and behavioral symptoms. This postimprovement decline in cognitive functioning has been previously reported in a substantial proportion of patient with TBI, and this can be mediated by emotional disturbances and the number of therapy hours in the rehabilitation facility (Millis et al., 2001; Ruff et al.,1991). Of particular relevance here is the role of an enriched environment provided to the patient once the hospital-based rehabilitation is over. Past evidence is indicative of the role of an enriched environment in fostering experience dependent neuroplasticity and thereby facilitating cognitive improvement. Understandably, a lack of environmental enrichment may lead to the weakening of the neural pathways and may consequently result in cognitive decline (Frasca, Tomaszczyk, McFadyen, \& Green, 2013). The factors associated with environmental enrichments may range from financial resources, social support, preparedness for transition from rehabilitation facility to home setup, and routines with cognitive, social, and physically stimulating activities (Frasca et al., 2013). The lack of cognitive stimulation in a small town in eastern India along with overprotective caregivers would have resulted in lack of environmental enrichment and finally led to decline in certain cognitive domains. Therefore, the patient was advised to continue home-based cognitive retraining and to come for EEG-NFT at their convenience.

After 3 months of home-based cognitive retraining, the patient received 20 sessions of EEG-NFT in phase II of neuropsychological rehabilitation. The NPA completed at the end of phase II revealed significant improvement in the domains of processing speed, response inhibition, visuospatial construction, and retention of verbal memory. The scores in these domains were above 15th percentile and thereby were out of the impaired range, which is a marked deviation from the trend observed throughout the previous NPAs. The VAS was also suggestive that the overall dysfunction decreased to $15 \%$, with significant gains in the domains of orientation, irritability, and sadness. There was also significant improvement in mood, a considerable reduction in irritability, greater motivation in attempting to recall information, and a higher sense of perceived autonomy. These findings need to be interpreted in light of the fact that symptom reduction and improvement across several cognitive, emotional, and behavioral domains has been seen after 2 years of the TBI. However, the findings from the present case add to the accumulated evidence that EEG-NFT may continue to enhance functioning long after the structural damage to the brain (Hammond, 2007). Improvement in the symptom reduction and enhanced cognitive functioning has serious implications in the quality of life (QoL) of a patient with TBI. The QoL in these patients reflect the life-altering changes due to the TBI that are interwoven with near-permanent physical, cognitive, and social sequelae of the syndrome. These findings are of value in light of Heinemann and Whiteneck's (1995) linear model of QoL, which 
postulates that the disability and impairment in numerous domains faced by patients with TBI translates into poor functioning which in turn leads to poor QoL.

Overall the results of EEG-NFT integrated neuropsychological rehabilitation in the present case are similar to the randomized controlled trial conducted by Cicerone et al. (2008). These authors reported that holistic neuropsychological rehabilitation was effective in enhancing the workrelated productivity, perceived QoL, and cognitive functioning. However, integration of EEG-NFT into neuropsychological rehabilitation program may help speed up the recovery process as it has the potential to alleviate cognitive as well as emotionalbehavioral problems. Moreover in patients with severe behavioral disturbances, the individual therapies may not be feasible. In such instances EEG-NFT can be used initially in the neuropsychological rehabilitation process. Other psychotherapeutic interventions can be initiated once the behavioral disturbances have reduced.

\section{Conclusion}

Severe TBI is associated with poor long-term outcomes and chronic disability. The overlay of cognitive and behavioral disturbances in these patients may further complicate the clinical scenario. The neuropsychological rehabilitation in these patients is challenging and requires simultaneous interventions to address the multitude of problems experienced by the patient and family. EEG-NFT is a promising intervention in rehabilitation after TBI. The present case study highlights that integrating EEG-NFT along with other psychotherapeutic interventions resulted in better gains through the processes of neuropsychological rehabilitation. Though robust studies are required to establish the effectiveness of EEG-NFT integrated neuropsychological rehabilitation, a case study proves to be of immense value in delineating the finer nuances of each intervention provided in the neuropsychological rehabilitation and its contribution in overall recovery.

\section{Author Disclosure}

This study was supported by the Indian Council of Medical Research (ICMR), New Delhi, India, through the research fellowship [No.3/1/3/2014/HRD /97(32803)].

\section{References}

Ahmed, S., Venigalla, H., Mekala, H. M., Dar, S., Hassan, M., \& Ayub, S. (2017). Traumatic brain injury and neuropsychiatric complications. Indian Journal of Psychological Medicine, 39(2), 114-121. https://doi.org/10.4103/0253-7176.203129

Albrecht, J. S., Barbour, L., Abariga, S. A., Rao, V., \& Perfetto, E. M. (2019). Risk of depression after traumatic brain injury in a large national sample. Journal of Neurotrauma, 36(2), 300307. https://doi.org/10.1089/neu.2017.5608

Bédard, M., Felteau, M., Marshall, S., Dubois, S., Gibbons, C., Klein, R., \& Weaver, B. (2012). Mindfulness-based cognitive therapy: Benefits in reducing depression following a traumatic brain injury. Advances in Mind-Body Medicine, 26(1), 14-20.

Benedictus, M. R., Spikman, J. M., \& van der Naalt, J. (2010). Cognitive and behavioral impairment in traumatic brain injury related to outcome and return to work. Archives of Physical Medicine and Rehabilitation, 91(9), 1436-1441. https://doi.org /10.1016/j.apmr.2010.06.019

Bennett, C. N., Gupta, R. K., Prabhakar, P., Christopher, R., Sampath, S., Thennarasu, K., \& Rajeswaran, J. (2018) Clinical and biochemical outcomes following EEG neurofeedback training in traumatic brain injury in the context of spontaneous recovery. Clinical EEG and Neuroscience, 49(6), 433-440. https://doi.org/10.1177/1550059417744899

Bennett, C. N., Rajeswaran, J., Sampath, S., \& Christopher, R (2013). The right to write: EEG neurofeedback training in frontal lobe agraphia-A case report. Journal of Neurotherapy, 17(3), 162-165. https://doi.org/10.1080 /10874208.2013.813174

Bennett, C. N., Sampath, S., Christopher, R., Thennarasu, K., \& Rajeswaran, J. (2017). Effect of electroencephalogram neurofeedback training on quality of life in patients with traumatic brain injury: In context of spontaneous recovery. Indian Journal of Neurotrauma, 14(02/03), 129-134. https://doi.org/10.1055/s-0038-1649280

Ben-Yishay, Y., \& Diller, L. (2011). Handbook of holistic neuropsychological rehabilitation: Outpatient rehabilitation of traumatic brain injury. New York, NY: Oxford University Press.

Blake, H. (2008). Caregiver stress in traumatic brain injury. International Journal of Therapy and Rehabilitation, 15(6), 263-271. https://doi.org/10.12968/ijtr.2008.15.6.29878

Burton, A. (2016). A key traumatic brain injury initiative in India. The Lancet Neurology, 15(10), 1011-1012. https://doi.org /10.1016/S1474-4422(16)30131-4

Byers, A. P. (1995). Neurofeedback therapy for a mild head injury. Journal of Neurotherapy, 1(1), 22-37. https://doi.org/10.1300 /J184v01n01 04

Cicerone, K. D., Goldin, Y., Ganci, K., Rosenbaum, A., Wethe, J. V., Langenbahn, D. M., ... Harley, J. P. (2019). Evidencebased cognitive rehabilitation: Systematic review of the literature from 2009 through 2014. Archives of Physical Medicine and Rehabilitation, 100(8), 1515-1533. https://doi.org/10.1016/j.apmr.2019.02.011

Cicerone, K. D., Mott, T., Azulay, J., Sharlow-Galella, M. A., Ellmo, W. J., Paradise, S., \& Friel, J. C. (2008). A randomized controlled trial of holistic neuropsychologic rehabilitation after traumatic brain injury. Archives of Physical Medicine and Rehabilitation, 89(12), 2239-2249. https://doi.org/10.1016 /j.apmr.2008.06.017

Cole, M. A., Muir, J. J., Gans, J. J., Shin, L. M., D'Esposito, M., Harel, B. T., \& Schembri, A. (2015). Simultaneous treatment of neurocognitive and psychiatric symptoms in veterans with post-traumatic stress disorder and history of mild traumatic brain injury: A pilot study of mindfulness-based stress reduction. Military Medicine, 180(9), 956-963. https://doi.org /10.7205/milmed-d-14-00581

Dewan, M. C., Rattani, A., Gupta, S., Baticulon, R. E., Hung, Y.C., Punchak, M., ... Park, K. B. (2018). Estimating the global 
incidence of traumatic brain injury. Journal of Neurosurgery, 130(4), 1080-1097. https://doi.org/10.3171/2017.10.jns17352

Dikmen, S. S., Corrigan, J. D., Levin, H. S., Machamer, J., Stiers, W., \& Weisskopf, M. G. (2009). Cognitive outcome following traumatic brain injury. The Journal of Head Trauma Rehabilitation, 24(6), 430-438. https://doi.org/10.1097 /htr.0b013e3181c133e9

Dikmen, S. S., Machamer, J. E., Powell, J. M., \& Temkin, N. R. (2003). Outcome 3 to 5 years after moderate to severe traumatic brain injury. Archives of Physical Medicine and Rehabilitation, 84(10), 1449-1457. https://doi.org/10.1016 /s0003-9993(03)00287-9

Doornhein, K., \& De Haan, E. H. F. (1998). Cognitive training for memory deficits in stroke patients. Neuropsychological Rehabilitation, 8(4), 393-400. https://doi.org/10.1080 1713755579

Foulkes, M. A., Eisenberg, H. M., Jane, J. A., Marmarou, A., \& Marshall, L. F. (1991). The Traumatic Coma Data Bank: Design, methods, and baseline characteristics. Journal of Neurosurgery, 75(Suppl.), S8-S13. https://doi.org/10.3171 /sup.1991.75.1s.00s8

Frasca, D., Tomaszczyk, J., McFadyen, B. J., \& Green, R. E. (2013). Traumatic brain injury and post-acute decline: What role does environmental enrichment play? A scoping review. Frontiers in Human Neuroscience, 7, 31. https://doi.org /10.3389/fnhum.2013.00031

Gupta, R. K., Afsar, M., Yadav, R. K., Shukla, D. P., \& Rajeswaran, J. (2020). Effect of EEG neurofeedback training in patients with moderate-severe traumatic brain injury: A clinical and electrophysiological outcome study. NeuroRegulation, $7(2), \quad 75-83 . \quad$ https://doi.org/10.15540 /nr.7.2.75

Hammond, D. C. (2007). Can LENS neurofeedback treat anosmia resulting from a head injury? Journal of Neurotherapy, 11(1), 57-62. https://doi.org/10.1300/J184v11n01 06

Heinemann, A. W., \& Whiteneck, G. G. (1995). Relationships among impairment, disability, handicap, and life satisfaction in persons with traumatic brain injury. The Journal of Head Trauma Rehabilitation, 10(4), 54-63. https://doi.org/10.1097 /00001199-199508000-00006

Keller, I. (2001). Neurofeedback therapy of attention deficits in patients with traumatic brain injury. Journal of Neurotherapy, 5(1-2), 19-32. https://doi.org/10.1300/J184v05n01_03

Kersel, D. A., Marsh, N. V., Havill, J. H., \& Sleigh, J. W. (2001). Neuropsychological functioning during the year following severe traumatic brain injury. Brain Injury, 15(4), 283-296. https://doi.org/10.1080/02699050010005887

Leclercq, M., Couillet, J., Azouvi, P., Marlier, N., Martin, Y., Strypstein, E., \& Rousseaux, M. (2000). Dual task performance after severe diffuse traumatic brain injury or vascular prefrontal damage. Journal of Clinical and Experimental Neuropsychology, 22(3), 339-350. https://doi.org/10.1076/1380-3395(200006)22:3;1-v;ft339

Madigan, N. K., DeLuca, J., Diamond, B. J., Tramontano, G., \& Averill, A. (2000). Speed of information processing in traumatic brain injury: Modality-specific factors. The Journal of Head Trauma Rehabilitation, 15(3), 943-956. https://doi.org/10.1097/00001199-200006000-00007

Majid, M. J., Lincoln, N. B., \& Weyman, N. (2000). Cognitive rehabilitation for memory deficits following stroke. Cochrane Database of Systematic Reviews, 3, CD002293. https://doi.org/10.1002/14651858.CD002293

May, G., Benson, R., Balon, R., \& Boutros, N. (2013). Neurofeedback and traumatic brain injury: A literature review. Annals of Clinical Psychiatry, 25(4), 289-296.

McAvinue, L., O’Keeffe, F., McMackin, D., \& Robertson, I. H. (2005). Impaired sustained attention and error awareness in traumatic brain injury: Implications for insight. Neuropsychological Rehabilitation, 15(5), 569-587. https://doi.org/10.1080/09602010443000119
Menon, D. K., Schwab, K., Wright, D. W., \& Maas, A. I. (2010). Position statement: Definition of traumatic brain injury. Archives of Physical Medicine and Rehabilitation, 91(11), 1637-1640. https://doi.org/10.1016/j.apmr.2010.05.017

Millis, S. R., Rosenthal, M., Novack, T. A., Sherer, M., Nick, T. G., Kreutzer, J. S., ... Ricker, J. H. (2001). Long-term neuropsychological outcome after traumatic brain injury. The Journal of Head Trauma Rehabilitation, 16(4), 343-355. https://doi.org/10.1097/00001199-200108000-00005

Munivenkatappa, A., Rajeswaran, J., Indira Devi, B., Bennet, N. \& Upadhyay, N. (2014). EEG Neurofeedback therapy: Can it attenuate brain changes in TBI? NeuroRehabilitation, 35(3), 481-484. https://doi.org/10.3233/nre-141140

Qadeer, A., Khalid, U., Amin, M., Murtaza, S., Khaliq, M. F., \& Shoaib, M. (2017). Caregiver's burden of the patients with traumatic brain injury. Cureus, 9(8), e1590. https://doi.org $/ 10.7759 /$ cureus. 1590

Rabinowitz, A. R., \& Levin, H. S. (2014). Cognitive sequelae of traumatic brain injury. Psychiatric Clinics of North America, 37(1), 1-11. https://doi.org/10.1016/j.psc.2013.11.004

Rajeswaran, J. (Ed.). (2012). Neuropsychological rehabilitation: Principles and applications. Oxford, UK: Newnes.

Rao, S. L., Subbakrishna, D. K., \& Gopukumar, K. (2004). NIMHANS neuropsychology battery manual. Bangalore, India: National Institute of Mental Health and Neurosciences.

Rao, S. L. (2004). Home-based cognitive retraining. Paper presented at the II Workshop on 369 Continuing Education in Neuropsychology. Bangalore, India: National Institute of Mental Health and Neurosciences.

Rassovsky, Y., Satz, P., Alfano, M. S., Light, R. K., Zaucha, K., McArthur, D. L., \& Hovda, D. (2006). Functional outcome in TBI II: Verbal memory and information processing speed mediators. Journal of Clinical and Experimental Neuropsychology, 28(4), 581-591. https://doi.org/10.1080 /13803390500434474

Reddy, R. P., Jamuna, N., Indira Devi, B., \& Thennarasu, K. (2009). Neurofeedback training to enhance learning and memory in patient with traumatic brain injury: A single case study. The Indian Journal of Neurotrauma, 6(1), 87-90. https://doi.org/10.1016/S0973-0508(09)80037-3

Reddy, R. P., Rajeswaran, J., Bhagavatula, I. D., \& Kandavel, T. (2014). Silent epidemic: The effects of neurofeedback on quality-of-life. Indian Journal of Psychological Medicine, 36(1), 40. https://doi.org/10.4103/0253-7176.127246

Reddy, R. P., Rajeswaran, J., Indira Devi, B., \& Kandavel, T. (2013). Neurofeedback training as an intervention in a silent epidemic: An Indian scenario. Journal of Neurotherapy, 17(4), 213-225. https://doi.org/10.1080/10874208.2013.847139

Reddy, R. P., Rajeswaran, J., Indira Devi, B., \& Kandavel, T. (2017). Cascade of traumatic brain injury: A correlational study of cognition, postconcussion symptoms, and quality of life. Indian Journal of Psychological Medicine, 39(1), 32-39. https://doi.org/10.4103/0253-7176.198940

Ruff, R. M., Young, D., Gautille, T., Marshall, L. F., Barth, J., Jane, J. A., ... Foulkes, M. A. (1991). Verbal learning deficits following severe head injury: Heterogeneity in recovery over 1 year. Journal of Neurosurgery, 75(Suppl.), S50-S58. https://doi.org/10.3171/sup.1991.75.1s.0s50

Scholten, A. C., Haagsma, J. A., Cnossen, M. C., Olff, M., van Beeck, E. F., \& Polinder, S. (2016). Prevalence of and risk factors for anxiety and depressive disorders after traumatic brain injury: A systematic review. Journal of Neurotrauma, 33(22), 1969-1994. https://doi.org/10.1089/neu.2015.4252

Serino, A., Ciaramelli, E., Di Santantonio, A., Malagù, S., Servadei, F., \& Làdavas, E. (2006). Central executive system impairment in traumatic brain injury. Brain Injury, 20(1), 2332. https://doi.org/10.1080/02699050500309627

Stéfan, A., \& Mathé, J.-F. (2016). What are the disruptive symptoms of behavioral disorders after traumatic brain injury? A systematic review leading to recommendations for good 
practices. Annals of Physical and Rehabilitation Medicine, 59(1), 5-17. https://doi.org/10.1016/j.rehab.2015.11.002

Sternbach, G. L. (2000). The Glasgow Coma Scale. The Journal of Emergency Medicine, 19(1), 67-71. https://doi.org/10.1016 /s0736-4679(00)00182-7

Thornton, K. (2000). Improvement/rehabilitation of memory functioning with neurotherapy/qEEG biofeedback. The Journal of Head Trauma Rehabilitation, 15(6), 1285-1296. https://doi.org/10.1097/00001199-200012000-00008

Tinius, T. P., \& Tinius, K. A. (2000). Changes after EEG biofeedback and cognitive retraining in adults with mild traumatic brain injury and attention deficit hyperactivity disorder. Journal of Neurotherapy, 4(2), 27-44. https://doi.org /10.1300/J184v04n02 05

von Bernhardi, R., Eugenín-von Bernhardi, L., \& Eugenín, J. (2017). What is neural plasticity? In R. von Bernhardi, J. Eugenín, \& K. Muller (Eds.), The Plastic Brain, Advances in Experimental Medicine and Biology (vol. 1015, pp. 1-15). Switzerland: Springer Nature. https://doi.org/10.1007/978-3319-62817-2_1
War, F. A., Jamuna, R., \& Arivazhagan, A. (2014). Cognitive and sexual functions in patients with traumatic brain injury. Asian Journal of Neurosurgery, 9(1), 29-32. https://doi.org/10.4103 11793-5482.131061

Wilson, B. A. (2008). Neuropsychological rehabilitation. Annual Review of Clinical Psychology, 4, 141-162. https://doi.org /10.1146/annurev.clinpsy.4.022007.141212

Yeates, K. O. (2010). Mild traumatic brain injury and postconcussive symptoms in children and adolescents. Journal of the International Neuropsychological Society, 16(6), 953-960. https://doi.org/10.1017/S1355617710000986

Zorcec, T., Demerdzieva, A., \& Pop-Jordanova, N. (2011). QEEG, brain rate, executive functions and neurofeedback training in patients with traumatic brain injury. Acta Informatica Medica, 19(1), 23-27.

Received: February 10, 2021

Accepted: March 8, 2021

Published: March 29, 2021 